\title{
A argumentação e o potencial metacognitivo de uma atividade experimental baseada na POA (Previsão- Observação-Argumentação)
}

The argumentation and metacognitive potential of an experimental activity based on poa (prediction-observation-argumentation)

Edilene Ferreira de Medeiros ${ }^{1}$ Marcia Gorette Lima da Silva² Solange Wagner Locatelli ${ }^{3}$

\section{Resumo}

Esta pesquisa apresenta incidentes metacognitivos de alunos do ensino médio ao vivenciar uma atividade experimental sobre fotossíntese e respiração baseada na PrevisãoObservação-Argumentação. A atividade inclui retomada dos conteúdos, elementos do argumento e guias de scripts. $O$ experimento selecionado objetivou aplicar conhecimentos de biologia em um contexto real e desenvolver habilidades argumentativas. Consistia em colocar uma planta em dois tubos contendo água com fenolftaleína, um permanecendo no escuro e o outro no claro. Foi solicitado aos alunos prever o que aconteceria com cada tubo e comparar com as mudanças na cor relacionadas ao pH. Participaram 8 alunos sendo a atividade realizada em grupos cooperativos promovendo discussão a partir da perspetiva de participação em práticas científicas. Concretamente foram analisados incidentes metacognitivos presentes no discurso dos alunos quando justificavam suas conclusões baseadas nos dados obtidos no experimento. Os resultados indicaram 4 categorias de incidentes metacognitivos: confirmação, monitoramento, mudança positiva e mudança negativa. Nos discursos mostraram maior habilidade para observar dados experimentais e justificar suas conclusões depois de confrontar suas previsões iniciais com conhecimento científico. No entanto, apresentaram dificuldades em argumentar suas explicações (incidente metacognitivo de mudança negativa). Sinalizamos que as atividades envolvendo a argumentação favoreceram a capacidade de autorregulação do processo de aprendizagem. Palavras chave: incidentes metacognitivos; Previsão-Observação-Argumentação; atividade experimental; fotossíntese; respiração.

\footnotetext{
1Universidade Federal do Rio Grande do Norte | lene.fmedeiros@gmail.com 2Universidade Federal do Rio Grande do Norte | marciaglsilva@yahoo.com.br 3Universidade Federal do ABC | solangeufabc@gmail.com
} 


\section{Abstract}

This research presents the metacognitive incidents of high school students when experiencing an experimental activity on photosynthesis and respiration based on Prediction-Observation-Argumentation. The activity includes resumption of content, argument elements, and script guides. The experiment was selected aiming to apply knowledge of biology in a real context and to develop argumentative abilities. It consisted of placing a plant in two tubes containing water with phenolphthalein, one remaining in a darkroom and another one in the clearing. Students were asked to predict what would happen to each tube and compare with changes in the color related to $\mathrm{pH}$. Eight students participated and the activity was carried out in cooperative groups, promoting discussion based on the perspective of participation in scientific practices. Specifically, metacognitive incidents were analyzed in the students' discourse when they justified their conclusions based on the data obtained in the experiment. The results indicated four categories of metacognitive incidents: confirmation, monitoring, positive change and negative change. In the discourses the students showed greater ability to observe experimental data and justify their conclusions after confronting their initial predictions with scientific knowledge. However, they present difficulties in arguing their explanations (metacognitive incident of negative change). We indicate that the activities promoting argumentation favor the selfregulation capacity of the learning process.

Keywords: metacognitive incidents; Prediction-Observation-Argumentation; experimental activity; photosynthesis; respiration.

\section{Introdução}

Qual a relevância do ensino da argumentação nas aulas de ciências? Em que medida se relaciona com a metacognição? Como favorecer a metacognição nas aulas de ciências? Estas e outras questões vêm sendo debatidas no campo da Didática das Ciências e, particularmente, nos interessa o potencial das atividades experimentais, enquanto ferramentas a favorecer a regulação do próprio processo de aprendizagem. É a partir destas inquietações iniciais que tentaremos delinear nossa linha de discurso neste texto à luz do referencial teórico e metodológico.

Argumentação e metacognição no ambiente escolar são temas que motivaram pesquisas recentes no campo da educação científica (CHIARO; AQUINO, 2017; GROTZER; MITTLEFEHLDT, 2012; OSBORNE, 2012; SÁNCHEZ-CASTAÑO; CASTAÑO-MEJÍA; TAMAYOALZATE, 2015; SCHWARZ, 2009; ZOHAR; DORI, 2012). De acordo com Sánchez-Castaño, Castaño-Mejía e Tamayo-Alzate (2015) essas duas categorias, geralmente, são investigadas separadamente: a primeira, com o objetivo de saber como o aluno argumenta, como o conhecimento é construído na sala de aula e como são as interações discursivas entre professores e alunos; e, a metacognição, em termos de caracterização dos processos relacionados à construção do conhecimento e ao controle sobre eles. Nesta perspectiva, há uma interface entre estas duas categorias a ser considerada durante os processos conscientes de autorregulação que "aprender a argumentar implica conscientemente e intencionalmente considerar certas estratégias metacognitivas que são encenadas no mesmo ato argumentativo" (SÁNCHEZ-CASTAÑO; CASTAÑO-MEJÍA; TAMAYO-ALZATE, 2015, p.1154, tradução nossa). 
Diante desse contexto, nosso trabalho tem como foco investigar a argumentação como promotora de processos metacognitivos, particularmente os incidentes metacognitivos presentes no discurso de estudantes do nível médio durante uma atividade experimental baseada na perspectiva POA (predizer-observar e argumentar) abordando o conteúdo de fotossíntese e respiração por meio de uma atividade experimental.

\section{Argumentação e metacognição: situando a relação com o Ensino de Ciências}

Neste estudo, partimos da premissa que a argumentação, enquanto atividade orientada, possibilita aos envolvidos, reflexões sobre seus próprios pensamentos em um nível não apenas cognitivo, mas também metacognitivo (CHIARO; AQUINO, 2017).

A literatura aponta estudos relacionados à utilização da argumentação em sala de aula como relevante para o desenvolvimento do pensamento crítico (DUSCHL; OSBORNE, 2002; GARCIA-MILÀ; ANDERSEN, 2008; IIMÉNEZ-ALEIXANDRE; BUGALLO-RODRÍGUEZ; DUSCHL, 2000; KELLY; DRUKER; CHEN, 1998; SASSERON et al., 2013; ZOHAR; NEMET, 2002), e em alguns contextos tem sido considerada prioridade no contexto escolar (CANDELA, 2001; ERDURAN, 2006; JIMÉNEZ-ALEIXANDRE, 2008; KUHN, 1993; OSBORNE; PATTERSON, 2011).

Mas, porque é importante que o aluno aprenda a argumentar?

Entre as contribuições da argumentação para o ensino de ciências, destacamos sua potencialidade para desenvolver o acesso aos processos cognitivos e metacognitivos, ou seja, proporcionar a oportunidade de aprender como aprender (ERDURAN; JIMÉNEZALEIXANDRE, 2008). Considera-se que, por meio dos discursos durante a explicitação do argumento, os processos de pensamento, normalmente pouco acessíveis na sala de aula, possam ser autorregulados, pois necessitam apoiar suas afirmações e avaliar distintas posições.

Há estudos que apontam esta relação, apesar das pesquisas no campo da metacognição serem relativamente recentes (GEORGHIADES, 2004; GOMES; ALMEIDA, 2016; PINTRICH, 2002). John Flavell, em 1976, introduz o termo em seu trabalho sobre o desenvolvimento cognitivo de crianças e, a partir de então, muitos pesquisadores passaram a investigar sua ação sobre a área educacional (COOK; KENNEDY; MCGUIRE, 2013; GONZÁLEZ; ESCUDERO, 2007; JAHANGARD; SOLTANI; ALINEJAD, 2016; LOCATELLI, 2016; RICKEY; STACY, 2000; THOMAS; ANDERSON; NASHON, 2008; ZOHAR; BARZILAI, 2013).

De forma simplificada, a metacognição pode ser entendida como o "pensar sobre o pensamento". Porém, quando nos aprofundamos nessa temática, esse conceito se torna mais complexo e passa a envolver outros atributos. Flavell (1976) definiu a metacognição como um conhecimento que um indivíduo pode ter a respeito do seu próprio processo cognitivo. Este, por sua vez, se refere a ideia da tomada de consciência por parte do indivíduo sobre seu próprio conhecimento (WHITE, 1988), podendo assim conhecer e controlar os mecanismos cognitivos referentes a processos de memória, atenção, compreensão etc. Ou seja, é possível analisar de forma intencional e consciente as decisões mentais mobilizadas para aprender um conteúdo ou resolver um problema, o que é fundamental no processo de aprendizagem. Isto implica em avaliar o que se sabe, planejar estratégias, enfim, favorecer a regulação do seu funcionamento intelectual (GONZÁLEZ, 1996; COSTA, 1984).

Nesta direção, a metacognição pode ser considerada a chave para uma aprendizagem mais profunda, durável e transferível, sendo dois pontos relevantes a saber: a consciência e 
o controle dos próprios pensamentos. A consciência dos próprios pensamentos implica em desenvolver a compreensão das ideias (ALEXANDER; JUDY, 1988) e o controle dos pensamentos reflete o impacto significativo em situações nas quais os estudantes estão envolvidos em resolução de problemas (FLAVELL, 1976). Para Rickey e Stacy (2000), a consciência de seus próprios pensamentos os ajuda a desenvolver a compreensão dos conceitos científicos e thes permite reconhecer quando suas ideias não são produtivas ou não podem ser conciliadas com dados (coletados ou fornecidos) ou ainda aquelas apresentadas por outros. Segundo esses autores, os estudantes que apresentam níveis mais elevados de atividade metacognitiva são mais propensos a refinar ideias ingênuas diante de resultados experimentais contraditórios. Além disso, antes de procurar explicações ou decidir sobre uma reflexão adicional para um dado tema, é necessário perceber de que maneira sua compreensão dos conceitos está incompleta.

De acordo com Linn e Songer (1993), a consciência de como vários conceitos e princípios se encaixa, também tende a conduzir a uma maior coerência do conhecimento. Em sala de aula a tomada de consciência dos próprios pensamentos pode ocorrer enquanto os alunos executam tarefas como ler, escrever e resolver problemas sejam eles teóricos ou experimentais, entre outras. Na mesma direção, Tobias e Everson (2002) consideram que o monitoramento prévio do aprendizado é um processo metacognitivo fundamental ou pré-requisito e, os aprendizes, que diferenciam com precisão o que foi aprendido anteriormente do que ainda têm a aprender, são mais capazes de concentrar a atenção e outros recursos cognitivos no material a ser aprendido. Para sustentar esta linha de raciocínio, esses autores apresentam os processos metacognitivos distribuídos hierarquicamente em uma pirâmide com quatro níveis (figura 1), na qual sua base é constituída pelo monitoramento do conhecimento, seguido da avaliação da aprendizagem, da capacidade de selecionar estratégias e do planejamento, no seu ápice.

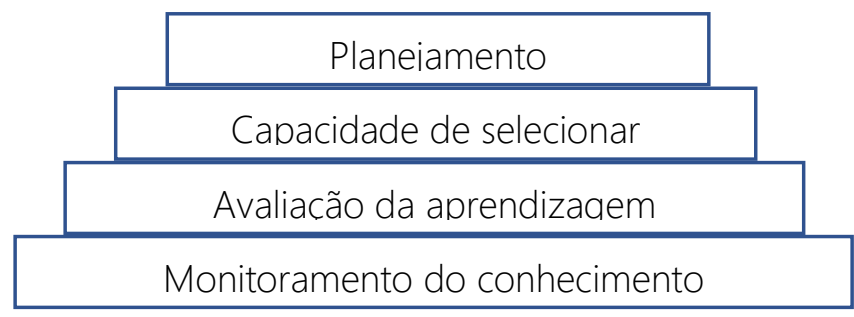

Figura 1: Níveis hierárquicos da Metacognição. Fonte: Tobias e Everson, 2002 (adaptação nossa)

A metacognição implica estar atento aos pontos fortes e fracos de nosso próprio funcionamento intelectual e aos tipos de erros de raciocínio que normalmente cometemos. Essa consciência e monitoramento nos ajudaria a explorar nossos pontos fortes, compensar as fragilidades e evitar erros mais comuns e contundentes (NICKERSON, 1984; BAKER, 1982) e, consequentemente a aprendizagem. Há estudos sinalizando a dificuldade de estudantes em se apropriar de algumas estratégias metacognitivas (COOK, KENNEDY, MCGUIRE, 2013; GONZÁLEZ; ESCUDERO, 2007; RICKEY; STACY, 2000) ou ainda buscam orientar os estudantes acerca da autorregulação da aprendizagem por meio de atividades visuais (LOCATELLI, 2016).

Em nossa abordagem buscamos revelar a relação entre argumentação e metacognição. Assumimos que ao argumentar o indivíduo expõe suas justificativas, as quais devem estar apoiadas em provas, e nesse movimento para construir um argumento 
cientificamente aceito, é levado a utilizar tanto sua capacidade cognitiva como metacognitiva. Nos apoiamos nas reflexões de Flavell (1987) sobre as propriedades que facilitam o desenvolvimento da metacognição ao apontar o desejo dos indivíduos em comunicar, explicar e justificar seu pensamento tanto para outras pessoas como para si mesmo e, no interesse em avaliar criticamente planos e pensamentos alternativos aos seus, quando estaria, consequentemente, envolvido em atividades de natureza metacognitiva. É exatamente a partir dessa interface da argumentação com o processo metacognitivo que se mostra relevante o planejamento de atividades em sala de aula (CHIARO; AQUINO, 2017; RICKEY; STACY, 2000; SMITH; METZ, 1996). Assim, diferentes estratégias podem ser utilizadas na sala de aula para auxiliar o desenvolvimento da metacognição, por exemplo, o uso de perguntas (COOK; KENNEDY; MCGUIRE, 2013; FLAVELL, 1976), a elaboração de mapas conceituais, as estratégias Predizer-Observar-Explicar (POE) e Modelar-ObservarRefletir-Explicar (MORE) (RICKEY; STACY, 2000).

Particularmente, com relação a POE, a etapa Predizer implica que o estudante exponha suas concepções iniciais sobre o que espera ocorrer no fenômeno (podendo ser um experimento). Na etapa Observação, pode realizar ou apenas observar o experimento em laboratório e, finalmente, na etapa Explicação elabora suas explicações. Esta etapa é interessante, na medida em que cria uma condição favorável ao estudante reexaminar suas ideias tentando conciliar/confrontar com aquelas expostas inicialmente com o que foi observado.

Locatelli e Arroio (2014b) sugerem uma modificação na POE com a adição de mais duas etapas metacognitivas com o objetivo de possibilitar, ainda mais, a autorregulação do processo, sendo elas o repensar e o reexplicar (POE-RR). Importante esclarecer que consideramos que explicar e argumentar são habilidades diferentes e, a falta de uma delimitação clara no planejamento da atividade pode levar o aluno a confundir explicação com argumentação (OSBORNE; PATTERSON, 2011; SASSERONet al., 2013). Nesta perspectiva, argumentar é a capacidade de avaliar os enunciados com base em provas, ou seja, reconhecer que as conclusões e enunciados científicos devem estar justificados, ou em outras palavras sustentados em provas; enquanto que explicar é a capacidade de expor as razões para a ocorrência de um determinado fato observado sem necessariamente fazer a conexão entre dado, justificativa e conclusão.

Com base nestas colocações, buscamos relacionar a argumentação como promotora de processos metacognitivos durante uma atividade experimental, sobre fotossíntese e respiração utilizando a estratégia $\mathrm{POA}^{4}$. Particularmente, investigamos os incidentes metacognitivos presentes no discurso dos alunos, que significam as "mudanças observáveis na cognição de um foco na tarefa ou interação social em um nível cognitivo para se concentrar em um aspecto da própria cognição ou emoção ou um foco na regulação e controle do pensamento" (LARKIN, 2009, p 152).

Locatelli e Arroio (2014a) em um estudo envolvendo isomeria geométrica com alunos do ensino médio, propuseram uma atividade de autorregulação dos conceitos. O objetivo era verificar o monitoramento metacognitivo dos estudantes acerca da construção inicial de conceitos em isomeria geométrica. Após análise do conteúdo das falas dos alunos, foram elaboradas quatro categorias para os incidentes metacognitivos observados, a saber:

\footnotetext{
${ }^{4}$ Esta proposta vem sendo desenvolvida no âmbito de uma pesquisa de mestrado (MEDEIROS, 2018, no prelo).
} 
confirmação, monitoramento, mudança positiva e mudança negativa. Assim, utilizaremos as mesmas categorias em nosso estudo, pois verificou-se que as mesmas são adequadas à presente análise. Vale salientar que os incidentes metacognitivos podem ser 'indicações' de manifestação de metacognição, termo adotado por estes autores, pois não há como ter certeza da presença desses incidentes, mas sim, estabelecer um indicativo de sua manifestação (LOCATELLI; ARROIO, 2014a).

\section{Metodologia}

Nossa pesquisa teve um enfoque qualitativo (BOGDAN; BIKLEN, 1994), do tipo estudo de caso (LÜDKE; ANDRÉ, 1986) sendo desenvolvida no Instituto Federal de Educação Ciência e Tecnologia do Rio Grande do Norte, Campus Natal Central (IFRN/CENAT), durante um minicurso intitulado "Argumentação científica através de uma atividade experimental: desvendando a fotossíntese" com a participação de 8 estudantes (17-18 anos) do $4^{\circ}$ ano do curso técnico de Manutenção e Suporte.

O tema foi escolhido devido sua relevância para o ensino de Biologia, aliado ao fato de ser considerado de natureza abstrata e de difícil compreensão por parte dos estudantes que, muitas vezes, considera a fotossíntese e a respiração como fenômenos inversos e sem relação de complementariedade (LABRACE; CALDERIA; BORTOLOZZI, 2009) ou ainda apresentam concepções alternativas (KAWASAKI; BIZZO, 2000; QUEIROZ; AZEVEDO, 1987; ZAGO et al., 2007). Do ponto de vista didático, procuramos recordar alguns conceitos científicos relacionados ao tema. Para possibilitar maior interação, recorremos ao trabalho em pequenos grupos (OSBORNE et al. 2001), utilizando estratégias como thinkaloud ${ }^{5}$ (BAKER, 2002) proporcionando oportunidades para que construam socialmente os significados e desenvolvam uma compreensão conceitual mais rica (KELLY; DRUKEN; CHEN, 1998).

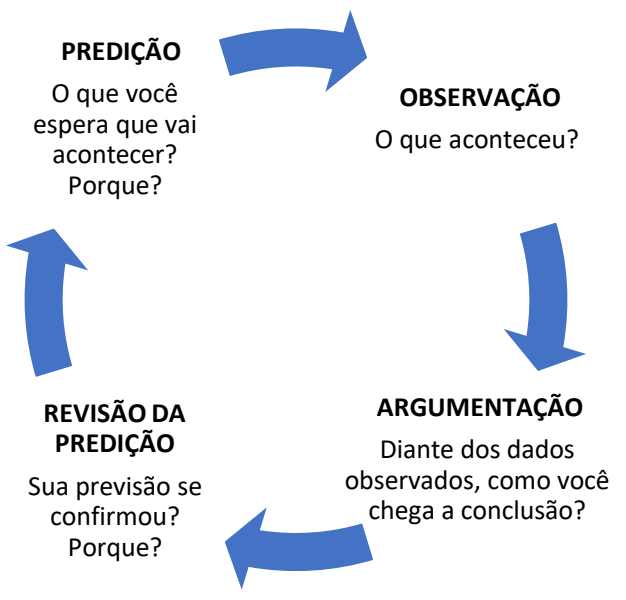

Figura 2: Etapas da atividade experimental baseada na estratégia POA.

\footnotetext{
5 "Pensar em voz alta" é uma técnica geralmente usada para tentar registrar o diálogo dos alunos e seu pensamento metacognitivo, bem como, favorece o envolvimento dos alunos no processo cognitivo (BAKER, 2002).
} 
A situação apresentada consistiu em uma atividade experimental estruturada com base na POA (Predizer-Observar-Argumentar), conforme a figura 2:

Para cada etapa (Predição e Observação) de investigação do comportamento dos tubos de ensaio no claro e no escuro, foram elaborados roteiros orientadores. Para a etapa da Argumentação, o roteiro abordava os seguintes pontos: Qual pergunta o experimento pretendia responder? Quais os dados coletados? Qual a justificativa para sua conclusão? Que conhecimento científico apoiava a sua justificativa? Qual a sua conclusão para o experimento? Nesta etapa os alunos foram encorajados a se engajar num processo de discussão para reivindicar, justificar, opor, questionar uns aos outros a fim de negociar e elaborar/reelaborar suas ideias, buscando compreender a concepção científica aceita, o que os leva a um exercício de metacognição.

Nossa análise se concentrou nas falas dos participantes durante a sessão de argumentação, as quais foram gravadas, transcritas e organizadas em episódios. Inicialmente, buscamos identificar os eventos metacognitivos dos não metacognitivos e, posteriormente, categorizá-los. Quer dizer, os indícios da presença de uma mudança observável na cognição de um foco na tarefa para o monitoramento, regulação e controle do pensamento. Para tal distinção nos apoiamos em Flavell (1976), o qual sugere que as estratégias cognitivas facilitam a conclusão de uma tarefa e do aprendizado enquanto que a metacognitiva monitora esse processo.

\section{Resultados e Discussão}

A atividade experimental adotada foi desenvolvida em um ambiente favorável ao processo de autorregulação (KIPNIS; HOFSTEIN, 2007). Esta consistiu em 2 conjuntos de tubos de ensaio, cada um contendo ramos da planta Elodea canadenses, água levemente alcalina e fenolftaleína (tubos B e D) e outro apenas com água levemente alcalina e fenolftaleína (tubos A e C). A figura 3 a seguir ilustra o resultado final após $24 \mathrm{~h}$.

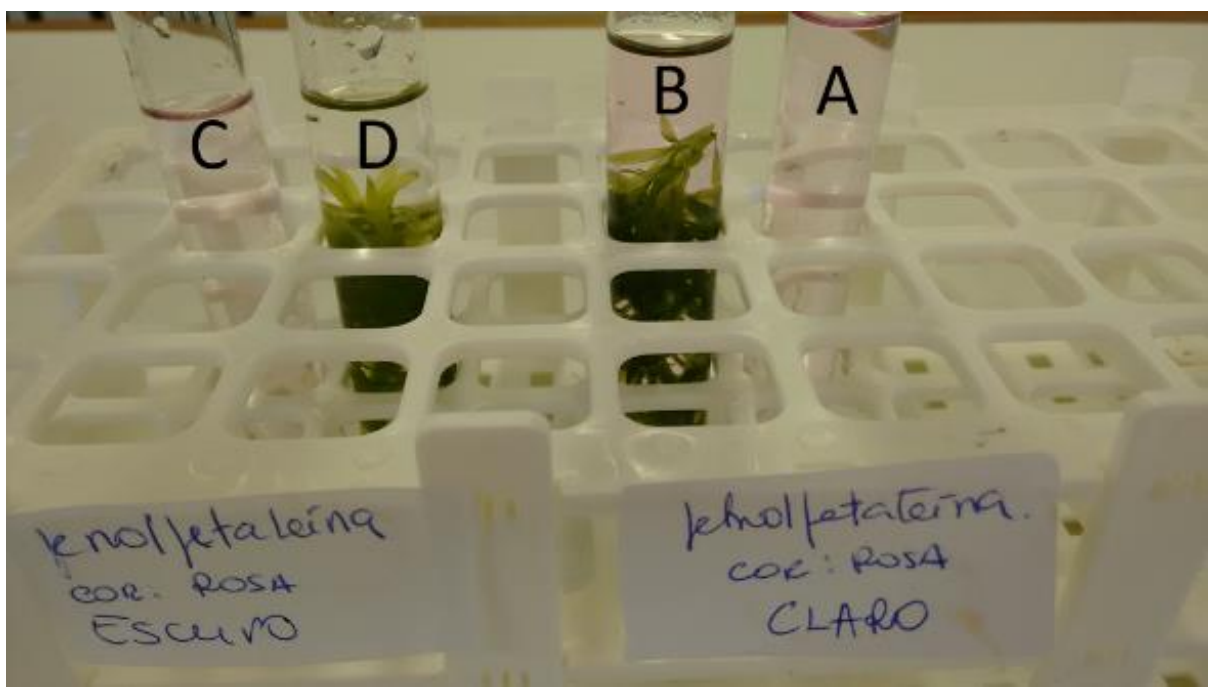

Figura 3: Resultado do experimento após 24 horas.

A tabela 1 apresenta os resultados observados e as respectivas explicações: 
Tabela 1: Síntese dos experimentos realizados.

\begin{tabular}{|c|c|c|c|c|}
\hline Tubo & A & B & C & $D$ \\
\hline $\begin{array}{l}\text { Condições } \\
\text { ambiente }\end{array}$ & \multicolumn{2}{|c|}{ Mantidos no ambiente claro por 24 horas } & \multicolumn{2}{|c|}{ Mantidos no ambiente escuro por 24 horas } \\
\hline Conteúdo & $\begin{array}{c}\text { Água e } \\
\text { fenolftaleína } \\
\text { (coloração rósea) }\end{array}$ & $\begin{array}{c}\text { Elodea canadenses, água e } \\
\text { fenolftaleína (coloração rósea) }\end{array}$ & $\begin{array}{c}\text { Água e } \\
\text { fenolftaleína } \\
\text { (coloração rósea) }\end{array}$ & $\begin{array}{c}\text { Elodea canadenses, água e } \\
\text { fenolftaleína (coloração } \\
\text { rósea) }\end{array}$ \\
\hline $\begin{array}{l}\text { Resultado } \\
\text { observado }\end{array}$ & $\begin{array}{l}\text { Não houve } \\
\text { mudança de } \\
\text { coloração. }\end{array}$ & $\begin{array}{l}\text { Intensificação de tonalidade da } \\
\text { cor rósea. }\end{array}$ & $\begin{array}{l}\text { Não houve } \\
\text { mudança de } \\
\text { coloração. }\end{array}$ & $\begin{array}{l}\text { Mudança de róseo para } \\
\text { incolor. }\end{array}$ \\
\hline Explicação & $\begin{array}{c}\text { A presença de } \\
\text { luz não interferiu. } \\
\text { Manteve a cor da } \\
\text { fenolftaleína em } \\
\text { meio levemente } \\
\text { alcalino. }\end{array}$ & $\begin{array}{c}\text { A intensificação de tonalidade } \\
\text { indica que o meio se tornou } \\
\text { um pouco mais alcalino. No } \\
\text { processo de respiração, a } \\
\text { planta libera } \mathrm{CO}_{2} \text {, porém, o } \\
\text { mesmo é consumido no } \\
\text { processo de fotossíntese. } \\
\text { Neste caso, diminuindo ainda } \\
\text { mais a concentração de íons } \\
\mathrm{H}_{3} \mathrm{O}^{+} \text {e tornando o meio mais } \\
\text { alcalino, sinalizado pela } \\
\text { intensificação da cor rosa. }\end{array}$ & $\begin{array}{l}\text { A ausência de luz } \\
\text { não interferiu. } \\
\text { Manteve a cor da } \\
\text { fenolftaleína em } \\
\text { meio levemente } \\
\text { alcalino. }\end{array}$ & $\begin{array}{l}\text { A mudança de coloração } \\
\text { indica variação do pH, } \\
\text { indicando que o meio está } \\
\text { ácido. O processo de } \\
\text { respiração da planta } \\
\text { liberou } \mathrm{CO}_{2} \text { que ao reagir } \\
\text { com a água formou ácido } \\
\text { carbônico }\left(\mathrm{H}_{2} \mathrm{CO}_{3}\right) \text { se } \\
\text { dissociando em } \\
\text { bicarbonato }\left(\mathrm{HCO}_{3}^{-}\right) \text {e íon } \\
\text { hidrônio }\left(\mathrm{H}_{3} \mathrm{O}^{+}\right) \text {. }\end{array}$ \\
\hline
\end{tabular}

Os participantes foram organizados em dois grupos. Em ambos mantiveram uma proximidade de incidentes metacognitivos (aproximadamente 26\% no grupo 1 e 30\% no grupo 2) e não metacognitivos (aproximadamente $74 \%$ no grupo 1 e $70 \%$ no grupo 2). 0 trecho a seguir exemplifica os incidentes:

A3: Onde você está vendo rosa? (incidente metacognitivo)

A4: Olhe aqui embaixo e olhe aqui em cima (se referindo à superfície do liquido no tubo de ensaio). É sutil, mas está (incidente não metacognitivo). A3: É sutil porque é inexistente (incidente não metacognitivo)

Neste episódio, consideramos como 'não metacognitivo' aquele incidente em que o aluno está apenas concluindo um raciocínio ou expressando uma explicação sobre o conteúdo do experimento e, como 'incidente metacognitivo' quando o aluno questiona (monitora) ou muda de foco cognitivo para o metacognitivo.

A partir da identificação dos incidentes metacognitivos, conforme já mencionado, adotamos a classificação de Locatelli e Arroio (2014a) em quatro grupos, a saber: confirmação, monitoramento, mudança positiva e mudança negativa, descritas a seguir.

Categoria confirmação: nesta categoria se observa a manifestação do incidente metacognitivo que confirma alguma ideia, algum processo de raciocínio ou conclusão. 0 recorte no diálogo a seguir exemplifica esta categoria.

Professor: $\mathrm{E}$, por que você conclui isso?

A2: Porque estava no escuro. Porque não tinha luz para ter fotossíntese (Confirmação) 
Neste recorte se observa o indício de confirmação no momento em que o aluno A2 expressa como chegou a sua conclusão e então ele confirma o raciocínio apresentado pelo grupo.

Categoria monitoramento: nesta categoria se observa a manifestação do incidente metacognitivo no qual há monitoramento de qualquer ideia ou raciocínio com o questionamento do que se está pensando. $O$ trecho a seguir ilustra esta categoria:

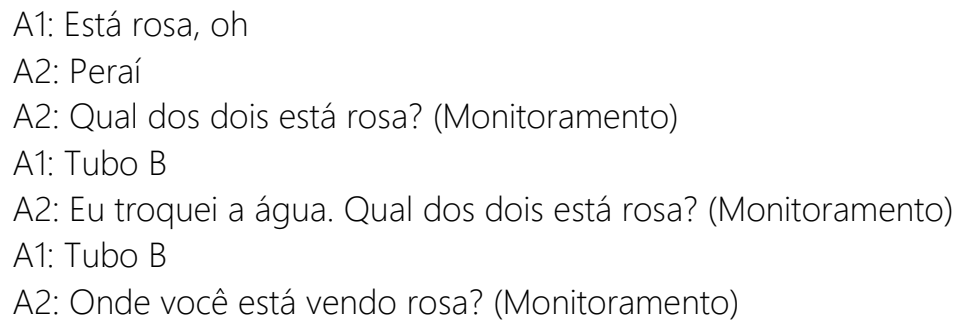

A manifestação desse incidente metacognitivo se dá a partir do debate entre as ideias, quando o aluno A2 não concorda com A1 sobre o resultado do experimento, questionando se realmente o tubo ficou cor de rosa.

Categoriamudança positiva (+): este incidente metacognitivo indica que houve uma mudança de ideia ou pensamento sobre algo, mas de forma positiva, ou seja, a ideia era incorreta, mas tornou-se uma resposta correta. $O$ trecho a seguir exemplifica esta categoria:

Professor: O meio está ácido. Então o que tornou o meio ácido?

A2: A liberação de H2 pela respiração. Não! é CO2. O CO2 se junta com a água e faz o ácido carbônico. (Mudança Positiva)

O indício do incidente metacognitivo 'Mudança Positiva' é observado a partir da discussão levantada pelo professor ao questionar o que tornou o meio ácido e, em resposta o aluno $A 2$, considera que o meio se tornou ácido em função da liberação de $\mathrm{H}_{2}$. Entretanto, esse aluno analisa seu raciocínio e reelabora sua resposta chegando a uma mais apropriada.

Categoriamudança negativa (-): nesta categoria o incidente metacognitivo é observado quando houve uma mudança de ideia ou pensamento sobre algo, mas de forma negativa, ou seja, indica que houve uma mudança na ideia, mas isso levou ao erro. $O$ trecho a seguir exemplifica esta categoria:

A3: Peraí. Esse é o do claro? Esse produziu oxigênio.

A2: Não, não, não é oxigênio é H+ é porque a fenolftaleína é só de $\mathrm{pH}$ e o lance é que um dos produtos da fotossíntese é H2 e o H2 deixa o meio ácido. E quando fica ácido não fica rosa fica incolor. Por isso esse está incolor (tubos no escuro). Na fase clara ele libera H2. Aí deixa isso claro (quer dizer incolor). (Mudança Negativa)

Pode-se inferir a indicação do incidente metacognitivo mudança negativa quando o aluno analisa a fala do colega ao tentar explicar a origem das bolhas, incorre em erro ao concluir que são provenientes do $\mathrm{H}^{+}$, bem como, confunde o íon $\mathrm{H}^{+}$com o $\mathrm{H}_{2}$. A tabela 2 reúne todos os incidentes metacognitivos identificados nas falas dos participantes: 
Tabela 2: Indícios de Incidentes Metacognitivos apresentados pelos grupos. Fonte: autoria própria.

\begin{tabular}{c|c|c}
\hline Categorias dos incidentes metacognitivos & Grupo 01 & Grupo 02 \\
\hline Confirmação & 5 & 6 \\
\hline Monitoramento & 8 & 8 \\
\hline Mudança positiva & 3 & 2 \\
\hline Mudança negativa & 1 & 1 \\
\hline
\end{tabular}

A partir da tabela 2, se observa que os incidentes metacognitivos de confirmação e monitoramento são os que mais aparecem nos discursos do alunado. Tal análise é corroborada com outros estudos (TOBIAS; EVERSON, 2002; LOCATELLI; ARROIO, 2014a). Para Tobias e Everson (2002) esses são procedimentos fundamentais, constituindo-se a base da pirâmide e, consequentemente, serão mais comuns. Estes, por sua vez, constituem prérequisitos para se alcançar estágios mais avançados da metacognição, isto é, os que estão no ápice da pirâmide (envolvendo avaliação, reavaliação e planejamento) por serem mais complexos e tendem a ocorrer em menor frequência, como percebemos em nosso trabalho em relação às mudanças positivas e negativas. Estes autores entendem o monitoramento do conhecimento como uma habilidade fundamental para o aprendizado nas escolas, quando bem constituído, pode promover o envolvimento dos alunos em atividades metacognitivas avançadas, como avaliar seu aprendizado de forma realista ou fazer planos para o controle efetivo dessa aprendizagem.

Os resultados também revelam a predominância, em ambos os grupos, dos incidentes de confirmação, monitoramento e mudanças positivas, podendo sinalizar que atividades experimentais baseadas na POA podem ter proporcionado a autorregulação da aprendizagem, já que permite ao aluno coletar, produzir e avaliar informações, podendo permitir que os aprendizes conheçam, controlem e autorregulem seu funcionamento intelectual. Dessa forma, a estratégia POA pode se constituir uma ferramenta eficiente para promover a metacognição em sala de aula. Principalmente no que diz respeito a etapa "argumentar" pois nesse processo o pensamento e o raciocínio, se tornam explícitos, pois os alunos expõem suas justificativas, as quais devem estar apoiadas em provas, e nesse movimento para construir um argumento cientificamente aceito, são levados a utilizar tanto sua capacidade cognitiva como metacognitiva (CHIARO, 2006; FLAVELL,1987).

$\mathrm{Na}$ etapa da argumentação, percebemos que apesar dos alunos apresentarem certa dificuldade em relação ao entendimento do conteúdo científico relativo a fotossíntese e respiração, aqui representado pelas mudanças negativas, foi possível observar que os participantes conseguiram elaborar um argumento bem estruturado (na perspectiva de Toulmin, 2006) incluindo dados, justificativa, conhecimento básico e refutação. Apesar de não ser o objeto deste artigo, é interessante ressaltar que um dos grupos chegou a apresentar uma refutação.

As falas a seguir exemplificam os argumentos elaborados pelos grupos:

Argumento do Grupo 01: Os tubos com elódea ficaram incolor e tubos sem elódea permaneceram rosa (dado), então ocorreu apenas respiração (conclusão), já que, ocorre a presença de CO2 no meio; o CO2 produzido na respiração deixa o meio ácido, pois se transforma em ácido carbônico 
e o meio ácido provoca a mudança de cor (implícito), bem como, não tinha luz para fazer fotossíntese (justificativa com conhecimento básico); a menos que, O CO2 tivesse sido utilizado (refutação).

Argumento do Grupo 02: Os dois tubos permaneceram rosa (ambiente claro). Os tubos não mudaram de cor (dados), então, ocorreu respiração e fotossíntese (conclusão), já que, a respiração libera CO2 entretanto como ocorre fotossíntese o $\mathrm{CO} 2$ produzido, ao invés de reagir com a água se transformando em ácido carbônico, é capturado para realizar a fotossíntese. Como não foi produzido ácido carbônico não ocorreu mudança no pH da água para que ela mudasse de cor (implícito) (justificativa com conhecimento básico).

A última etapa da atividade consista em rever as previsões iniciais. Observamos que os alunos foram capazes de perceber inconsistências em suas hipóteses e, assim reelaborá-las. Os trechos a seguir ilustram este monitoramento:

Grupo 1:

Previsão inicial: $\mathrm{O}$ tubo A se mantém rosa e o tubo B ficará incolor, pois, no ambiente claro o tubo B libera $\mathrm{H} 2$ e o tubo A não. O tubo $C$ e $D$ se mantém rosa pois, no ambiente escuro não ocorrerá fotossíntese e sim a respiração e o produto desta tornará o meio ácido.

Revisão da previsão: Nossa previsão não se manteve, pois, a predição envolvia a liberação de $\mathrm{H} 2$ no ambiente claro, porém, foi descoberto que o H2 é utilizado para produzir glicose. A previsão do tubo no ambiente escuro também não se concretizou pois, achamos que o meio se manteria básico.

Grupo 2:

Previsão inicial: No tubo A não acontece nada e no tubo B mesmo acontecendo a fotossíntese a planta vai liberar $\mathrm{O} 2$, mas não altera a cor da água pois, o $\mathrm{O} 2$ tem em todo lugar. Isto acontece devido a luz no ambiente claro. No tubo C não vai acontecer nada, não vai perder a cor rosa. E, no tubo D, provavelmente, muda de cor devido ao indicador de glicose.

Revisão da previsão: Nos tubos A e B a predição se confirmou, embora a justificativa da predição não foi exata. Nos tubos C e D a predição se confirmou, embora a justificativa não foi exata.

Observa-se pelos exemplos acima que os participantes, após as atividades desenvolvidas, foram capazes de rever seus argumentos explicativos envolvendo justificativas baseadas no conhecimento científico.

\section{Considerações Finais}

Em linhas gerais, foi possível sinalizar o papel que a argumentação desempenha em favorecer o processo metacognitivo. Tal posição se apoia em outros autores (citados anteriormente) ao discutir que os processos de reflexão metacognitiva podem ocorrer durante o ato de argumentar, isto é, favorece a um desenvolvimento gradual de ter consciência e controlar tanto o processo argumentativo como o metacognitivo ao qual é vinculado. 
Foi possível identificar as quatro categorias de incidentes metacognitivos, a saber: confirmação, monitoramento, mudança positiva e mudança negativa. Dentre elas, a de maior ocorrência foi o de monitoramento, o que pode revelar que a estratégia favoreceu o processo metacognitivo nos alunos, provavelmente devido: (1) a forma como estruturamos a atividade, proporcionando etapas bem direcionadas por meio de roteiros orientadores; (2) a organização dos alunos em grupos como potenciais para promover a interação, o engajamento e a reflexão coletiva de experiências de aprendizagem; (3) a participação do professor ao regular as ideias dos alunos orientando-osa refletiram a cerca das suas próprias ideias no intuito de redirecionar erros e inconsistências, favorecendo o processo de construção e reconstrução dos conceitos científicos pelos alunos.

Por outro lado, a ocorrência do incidente de 'mudança negativa' pode revelar, por exemplo, alguma dificuldade em relacionar com conhecimento científico (sobre fotossíntese e a respiração) ou até revelar suas concepções alternativas a respeito do tema e ainda, expor erros no raciocínio o que também é importante para redirecionar o raciocínio dos estudantes.

Como potencial continuidade deste estudo, dois aspectos são fundamentais, o planejamento de atividades com esta finalidade constitui um aspecto a ser mais explorado, a introdução de uma estratégia metavisual como etapa preliminar para iniciar a construção de conceitos, que podem ser importantes na compreensão submicroscópica dos fenômenos e, consequentemente na modelagem. Outro aspecto, de acordo com Larkin (2009), refere-se às possíveis interações do grupo com o desenvolvimento cognitivo individual. Do ponto de vista metodológico, os dados observacionais podem ser triangulados com outras análises de dados, em uma abordagem de estudo de caso. A complexidade dos perfis metacognitivos sociais sugere que sua abordagem na sala de aula pode resultar frutífera tanto no desenvolvimento de teorias de metacognição que levam em conta fatores sociais quanto na orientação didático-pedagógica.

\section{Referências}

ALEXANDER, P. A.; JUDY, J. E.The interaction of the domain specific and strategic knowledge in academic performance. Review of Educational Research, v. 58, p. 375-404, 1988.

BAKER, L. An Evaluation of the role of metacognitive deficits in learning disabilities.Topics in Learning and Learning Disabilities, v.2, n. 1, p. 27-34, 1982.

BAKER, L. Metacognition in comprehension instruction. In: BLOCK, C. C.; PRESLEY, M. (eds.). Comprehension instruction: research based best practices. New York: Guilford, p.77-95, 2002.

BOGDAN, R.; BIKLEN, S. Investigação qualitativa em educação. Uma introdução à teoria e aos métodos. Porto: Porto Editora, 1994.

CANDELA, A. Cienciaenel aula. Los alumnos entre laargumentación y el consenso. México, D. F.: Paidós, 2001.

CHIARO, S.Argumentação em sala de aula: um caminho para o desenvolvimento da autorregulação do pensamento. 2006. 94 f. Tese (Doutorado em Psicologia) - Universidade Federal de Pernambuco. CFCH. Psicologia Cognitiva, 2006. 
CHIARO, S.; AQUINO, K. A. S. Argumentação na sala de aula e seu potencial metacognitivo como caminho para um enfoque CTS no ensino de química: uma proposta analítica. Educação\&Pesquisa, São Paulo, v. 43, n. 2, p. 411-426, 2017.

COOK, E.; KENNEDY, E.; McGUIRE, S. Y. Effect of teaching metacognitive learning strategies on performance in general chemistry courses. Journal Chemical Education Research, v. 90, p. 961-967, 2013.

COSTA, A. L. Mediating the metacognitive (Mimeo) nov. 1984. Educationalleadership. Disponível em: <http://www.ascd.org/ASCD/pdf/journals/ed_lead/el_198411_costa.pdf>. Acesso em 16/12/2017.

DUSCHL, R. A.; OSBORNE, J. Supporting and promoting argumentation discourse. Studies in Science Education, v. 3, p. 39-72, 2002.

ERDURAN, S. Promoting ideas, evidence and argument in initial science teacher training.School Science Review, v.87, n. 321, p. 45-50, 2006.

ERDURAN, S.; JIMÉNEZ-ALEIXANDRE, M. P. Argumentation in science education: perspectives from classroom-based research, Dordrecht: Springer, 2008.

FLAVELL, J. H. Metacognitive aspects of problem solving. In: RESNICK, L.B. (Orgs.). The nature of intelligence. Hillsdale, N.Y.: Erlbaum, p. 231-235, 1976.

FLAVELL, J. H. Speculations about the nature and development of metacognition. In: F. WEINERT; R. KLUWE (eds.). Metacognition, motivation and understanding. Hillsdale, NJ: Erlbaum, p. 21-29, 1987.

GARCIA-MILA, M.; ANDERSEN, C. Cognitive Foundations of Learning Argumentation. In: ERDURAN, S.; JIMENEZ-ALEIXANDRE. P. M. (eds.). Argumentation in Science Education: Perspectives from classroom-based research. Dordrecht: Springer, p.29-45, 2008.

GEORGHIADES, P. From the general to the situated: Three decades of metacognition. InternationalJournalof Science Education, v. 26, p. 365-383, 2004.

GOMES, A. S.; ALMEIDA, A. C. P. Letramento científico e consciência metacognitiva de grupos de professores em formação inicial e continuada: um estudo exploratório. Amazônia: Revista de Educação em Ciências e Matemática, v. 12, n. 24, p. 53-76, 2016.

GONZÁLEZ, F. E. Acerca de lametacognición. Paradigma, v. 17, n. 1, 1996. Disponível em: $<$ http:// cidipmar.fundacite.arg.gov.ve/doc/paradigma96/doc5.htm>. Acesso em: 16/12/2017.

GONZÁLEZ, S.; ESCUDERO, C. En busca de laautonomía a través de lasactividades de cognición y de metacogniciónenCiencias. Revista Electrónica de Enseñanza de lasCiencias, v. 6, n. 2, p. 310-330, 2007.

GROTZER, T.; MITTLEFEHLDT, S.The role of metacognition in students understanding and transfer of explanatory structures in science. In: ZOHAR, A.; DORI, Y. J. (eds.). Metacognition in Science Education. Trends in current research, contemporary trends and issues in science education, New York: Springer, p.79-99, 2012.

JAHANGARD, Z; SOLTANI, A.; ALINEJAD, M. Exploring the relationship between metacognition and attitudes towards science of senior secondary students through a 
structural equation modeling analysis.Journal of Baltic Science Education, v.15, n. 3, p.34034, 2016.

JIMÉNEZ-ALEIXANDRE, M. P. Designing argumentation learning environments. In: ERDURAN, S.; JIMÉNEZ-ALEIXANDRE, M. P. (eds.). Argumentation in Science Education: perspectives from classroom-based research, Dordrecht: Springer, p.91-115, 2008.

JIMÉNEZ-ALEIXANDRE, M.P.; BUGALLO-RODRÍGUEZ, A.; DUSCHL, R. A. "Doing the lesson" or "doing science": argument in high school genetics". Science Education, v. 84, n. 20, p. 757-792, 2000

KAWASAKI, C. S.; BIZZO, N. M. V. Fotossíntese um Tema para o Ensino de Ciências? Conceitos Científicos em Destaque. Revista Química Nova na Escola, n. 12, p.24-29, 2000.

KELLY, G. J.; DRUKER, S.; CHEN, C. Students' reasoning about electricity: combining performance assessment with argumentation analysis. International Journal of Science Education, v. 20, n. 7, p.849-871, 1998.

KIPNIS, M.; HOFSTEIN, A.The inquiry laboratory as a source for development of metacognitive skills. International Journal of Science and Mathematics Education, v. 6, p. 601627, 2007.

$\mathrm{KUHN}, \mathrm{D}$. Science as argument: implications for teaching and learning science thinking. Science Education, v. 77, n. 3, p. 319-337, 1993.

LABRACE, E. C.; CALDEIRA, A. M. A.; BORTOLOZZI, J. A Atividade Prática no Ensino de Biologia: uma possibilidade de unir motivação, cognição e interação. In: CALDEIRA, A. M. A. (org.). Ensino de Ciências e Matemática II: Temas sobre a Formação de Conceitos. São Paulo: Editora, UNESP, p. 91-106, 2009.

LARKIN, S. Socially Mediated Metacognition and Learning to Write. Thinking skills and creativity, v. 4, p.149-159, 2009.

LINN, M. C.; SONGER, N. B. How do students make sense of science? Merrill-Palmer Quarterly, v.39, n.1, p. 47-73, 1993.

LOCATELLI, S.W. Relação existente entre metavisualização e as representações simbólica e submicro na elaboração de atividade em química. 2016. 311f. Tese (Doutorado em Ciências) - Universidade de São Paulo, São Paulo, 2016.

LOCATELLI, S.; ARROIO, A. Designing molecules and thinking about them: metavisual skill in the teaching of Geometrical Isomerism. Revista Brasileira do Ensino de Química. v.6, n.1 e n.2, p.99-112, 2011.

LOCATELLI, S.; ARROIO.The monitoring of an introductory class on geometrical isomerism by metavisual incidents.Journal of Science Education, v. 15, n. 2, p. 62-65, 2014 a.

LOCATELLI, S.; ARROIO.Metavisual strategy assisting the learning of initial concepts of electrochemistry.Natural Science Education, v.1, n.39, p.14-24, 2014 b.

LÜDKE, M.; ANDRÉ, A. D. E. M. Pesquisa em educação: abordagens qualitativas. São Paulo: Editora Pedagógica e Universitária, 1986.

MEDEIROS, E. F. Desenvolvendo Habilidades Argumentativas em Aulas de Biologia: Uma Atividade Experimental Baseada na Perspectiva POA (Predizer, Observar e Argumentar). 
2018. 122f. Dissertação (Mestrado em Ensino de Ciências Naturais e Matemática) Universidade Federal do Rio Grande do Norte -UFRN, Natal, 2018. No prelo.

NICKERSON, R. Kinds of thinking taught in currents programs. Educational Leadership. v. 42, n.1, p. 26-36, 1984.

OSBORNE, J. The role of Argument: Learning how to learn in School Science. In: FRASER, B. J; TOBIN, K.; MCROBBIE, C. (eds.). Second International Handbook of Science Education. New York: Springer, p. 933-949, 2012.

OSBORNE, J. F.; PATTERSON, A. Scientific argument and explanation: A necessary distinction? Science Education, v. 95, n. 4, p. 627-638, 2011.

OSBORNE, J. et al. Enhancing the quality of argument in school science. School science review, v. 82, n. 301, p. 63-70, 2001.

PINTRICH, P. R. The role of metacognitive knowledge in learning, teaching and assessing.TheoryintoPractice, v. 4, n. 4, p. 219-225, 2002.

QUEIROZ, G.; AZEVEDO, C. A. A ciência alternativa do senso comum e o treinamento de professores. Caderno Catarinense de Ensino de Física, Florianópolis, v. 4 n.1, p.7-16, 1987.

RICKEY, D.; STACY, A. M.The role of metacognition in learning chemistry.Journal of Chemical Education, v. 77, n.7, p. 915-920, 2000.

SÁNCHEZ-CASTAÑO, J. A.; CASTAÑO-MEJÍA, O. Y.; TAMAYO-ALZATE, O. E. La argumentaciónmetacognitivaenel aula de ciencias. Revista Latinoamericana de CienciasSociales, Niñez y Juventud, v.13, n.2, p. 1153-1168, 2015.

SASSERON, L. H. et al. Situações Argumentativas em Sala de Aula: a dualidade argumentoexplicação. In: IX CONGRES INTERNACIONAL SOBRE INVESTIGACIÓN EN DIDÁCTICA DE LAS CIENCIAS. Girona, p. 3233-3236, 2013.

SCHWARZ, B. B. Argumentation in Learning. In: MULLER, M. N.; PERRET-CLERMONT, A. N (eds.). Argumentation and Education.Theoretical foundations and practice.Breinigsville: Springer, p. 91-126, 2009.

SMITH K. J.; METZ P. A. Evaluating student understanding of solution chemistry through microscopic representations, Journal of Chemical Education, v. 73, p. 233-235, 1996.

THOMAS, G.; ANDERSON, D.; NASHON, S. Development of an Instrument Designed to Investigate Elements of Science Students' Metacognition, Self-Efficacy and Learning Processes: The SEMLI-S. International Journal of Science Education, v. 30, n. 13, p. 1701-1724, 2008.

TOBIAS, S; EVERSON, H. T. Knowing what you know and what you don't: further research on metacognitive knowledge monitoring. College Board Research Report, New York: College Entrance Examination Board, 2002.

TOULMIN, S. E. Os Usos do Argumento. Trad. Reinaldo Guarany e Marcelo Brandão Cipolla. 2 Ed. São Paulo: Martins Fontes, 2006.

WHITE, R. T. Metacognition. In: KEEVE, J. P. (ed.). Educational Research, Methodology and measurement: an international Handbook. Oxford: Pergamon, p. 70-75, 1988. 
ZAGO et al. Fotossíntese: Concepções dos Alunos do Ensino Médio de Itumbiara-GO e Buriti-Alegre-GO. RevistaBrasileira de Biociências, Porto Alegre, v. 5, p. 780-782, 2007.

ZOHAR A.; BARZILAI, S. A review of research on metacognition in science education: current and future directions. Studies in Science Education, v.49, n. 2, p.121-169, 2013.

ZOHAR, A.; DORI, Y. J. Introduction. In: ZOHAR, A.; DORI, Y. J. (eds.). Metacognition in Science Education. Breinigsville: Springer, p. 1-19, 2012.

ZOHAR, A.; NEMET, F. Fostering students' knowledge and argumentation skills through dilemmas in human genetics.Journal of Research in Science Teaching, v. 39, n.1, p.35-62. 2002. 